\title{
Decoherence and the measurement problem
}

\section{Herve Zwirn* \\ UFR de Physique (Université Paris 7) \& CMLA (ENS Cachan) \& IHPST (CNRS \& Université Paris 1)} CMLA, ENS Cachan, 61 avenue du Président Wilson, 94235 Cachan Cedex, France.

herve.zwirn@gmail.com

Abstract

The problem of measurement taken at face value shows clearly that there is an inconsistency inside the quantum formalism. The phenomenon of decoherence is often presented as a solution to it. A widely debated question is to decide between two different interpretations. The first one is to consider that the decoherence process has the effect to actually project a superposed state into one of its classically interpretable component, hence doing the same job as the reduction postulate. For the second one, decoherence is only a way to show why no macroscopic superposed state can be observed and so, to explain the classical appearance of the macroscopic world, while the quantum entanglement between the system, the apparatus and the environment never disappears. In this case, explaining why only one single definite outcome is observed remains to do. In this paper, we examine arguments for and against both interpretations and defend a position according to which the outcome that is observed is relative to the observer in close parallel to the Everett interpretation.

Frontiers of Fundamental Physics 14

FFP14

15-18 July 2014

Aix Marseille University (AMU) Saint-Charles Campus, Marseille

\section{*Speaker}




\section{The measurement problem}

As is well known the measurement problem comes from the fact that inside the quantum formalism, there are two contradictory postulates for computing the evolution of the state of a system. The first one is the Schrödinger equation: $i \hbar \frac{d|\Psi\rangle}{d t}=H|\Psi\rangle$ which is supposed to be used for an isolated system when no measurement is performed on it. The second one is the reduction postulate which says that when a measurement of a certain observable $A$ is made on a system which is initially in a state that is a superposition of eingenstates of $\mathrm{A},|\Psi\rangle=\sum c_{i}\left|\varphi_{i}\right\rangle$, then after the measurement, if the result is the eigenvalue of $A \lambda_{\mathrm{k}}$, the state $|\Psi\rangle$ is projected onto the eigenvector $\left|\varphi_{k}\right\rangle$ linked to this eigenvalue $\sum c_{i}\left|\varphi_{i}\right\rangle \rightarrow\left|\varphi_{k}\right\rangle$ or onto the sub-space of the Hilbert space that is spanned by the eigenvectors linked to it, if $\lambda_{\mathrm{k}}$ is degenerated.

It is of fundamental importance to realize that these two ways of describing the change of the state of a system are not at all compatible. The Schrödinger equation describes a linear and unitary process while the reduction postulate is neither linear nor unitary. That means that there is a priori no way one can get a reduction of the state through the Schrödinger equation.

That should not come as a problem if it was possible to give a clear and not ambiguous definition of what a measurement is. In this case, we would have two well separated situations, a first one when no measurement is made on the system and a second one when a measurement is made. In the first case, we should apply the Schrödinger equation and in the second one, the reduction postulate. It is nevertheless worth noticing that there is a difference in the way it is possible to use these two rules. The Schrödinger equation can be used without any further knowledge on the system. All is necessary to compute the future state at an arbitrary time is the initial state $|\Psi\rangle$ and the Hamiltonian $\mathrm{H}$ of the system. Using the reduction postulate is more demanding as it requires the knowledge of the result provided by the measurement. That could seem harmless as it is the case for example in classical statistical physics when one has only a probability distribution on the possible states of the system and when one updates the state when learning in which one of the possible states the system really is. But in quantum physics, this statistical interpretation is totally ruled out and hence, the very fact that the knowledge of the result is necessary lies at the core of the problem of the impossibility to state the quantum formalism without any reference to an observer (whatever at this stage an observer could be).

Now the problem is that it is impossible to define clearly what a measurement is! What is at stake is a definition that could be regarded as "strongly objective" in the meaning that d'Espagnat gave to this term [1] (i.e. without any mention to a human observer). Indeed, the Copenhagen interpretation, mainly proposed by Bohr, says that a measurement is an interaction between the system and a macroscopic classical apparatus ${ }^{1}$. Since a physicist using a macroscopic apparatus for measuring a physical property on a system perfectly knows what he is doing, there is no ambiguity and the reduction postulate must be applied. Even if this point of view is generally working in practice, it leaves open the question of knowing what a macroscopic apparatus is. The distinction micro / macro is not a sharp one and assuming naively that a macroscopic system behaves classically is problematic since we know of many macroscopic systems showing a quantum behavior (super conductivity, super fluidity etc.). Another difficulty with this view is that the quantum formalism is assumed to be universally valid and should be applied to any physical system, whether microscopic or macroscopic. A careful analysis of Bohr's position shows nevertheless that what he had in mind was not that the classical behavior of the apparatus was

\footnotetext{
${ }^{1}$ It even goes as far as saying that the property that is measured belongs not to the system itself but to the whole composed of the system plus the apparatus.
} 
directly linked to its macroscopic aspect but that it was linked to the use that the observer wanted to make of it. If the observer wants to make a measurement on it through another apparatus, it has a quantum behavior. If it is used to make an observation on another system, it has a classical behavior $^{2}$. In this case, it is clear that the role of a human observer in the definition of the measurement process can't be avoided and that this definition can't be considered as "strongly objective".

The notorious analysis given by von Neumann of a measurement process seen as an interaction between the system and an apparatus shows that two opposite and irreconcilable descriptions seem equally valid.

Assume that a measurement of a certain observable is made and that $\left\{\left|\varphi_{i}\right\rangle\right\}$ is a basis of the Hilbert space built from the eigenvectors of this observable associated with the associated eigenvalues $\lambda_{i}$ (that for the sake of simplicity, we assume to be non-degenerated). Let the system $\mathrm{S}$ be in a state $\left|\Psi_{S}\right\rangle=\sum c_{i}\left|\varphi_{i}\right\rangle$ and an apparatus $\mathrm{A}$ in the initial state $\left|A_{0}\right\rangle$. Then, before they interact, the state of the system - apparatus is the tensorial product:

$$
\left|\Psi_{S A}\right\rangle=\left|\Psi_{S}\right\rangle \otimes\left|A_{0}\right\rangle=\sum c_{i}\left|\varphi_{i}\right\rangle \otimes\left|A_{0}\right\rangle
$$

The interaction between $\mathrm{S}$ and $\mathrm{A}$ is done through a Hamiltonian $\mathrm{H}^{\mathrm{AS}}$ operating during a short time. It is assumed that the apparatus is built in such a way that if the system is in the state $\left|\varphi_{i}\right\rangle$, the apparatus will be in the state $\left|A_{i}\right\rangle$ after the measurement whatever its initial state. Now, there are two ways to describe the process.

The first one is to consider that the system-apparatus is an isolated global system on which no measurement is made and to use the Schrodinger equation. This gives:

$$
\left|\Psi_{S A}\right\rangle=\sum c_{i}\left|\varphi_{i}\right\rangle \otimes\left|A_{0}\right\rangle \rightarrow \sum c_{i}\left|\varphi_{i}\right\rangle \otimes\left|A_{i}\right\rangle
$$

The second one is to consider that a measurement is made on the system $\mathrm{S}$ and that a value $\lambda_{\mathrm{k}}$ is found. The reduction postulate gives:

$$
\left|\Psi_{S}\right\rangle=\sum c_{i}\left|\varphi_{i}\right\rangle \rightarrow\left|\varphi_{k}\right\rangle \text { and }\left|A_{0}\right\rangle \rightarrow\left|A_{k}\right\rangle
$$

Both description seem equally valid though they lead to totally different states. Equation (2) shows that the system and the apparatus are at the end in an entangled state. In particular, this is to be interpreted as if the apparatus was in a state which is a superposition of states linked to different possible results of the measurement ${ }^{3}$. For example, if the system is a spin $1 / 2$ particle and the apparatus a detector with a needle such that a spin up along Oz leads to a position of the needle pointing up (and a spin down, a position of the needle pointing down) then equation (2) leads to a state of the needle that is a superposition of positions up and down. Of course, no such macroscopic superposition has ever been observed. If we add another system (even a cat) to the initial system and the apparatus, it becomes entangled as well with the first two. This is the core of the celebrated Schrodinger's cat argument.

\section{Firstly proposed solutions}

Faced to what seems a real inconsistency inside the quantum formalism, physicists have proposed many solutions. A first category of solutions considers that even if the quantum formalism has a

\footnotetext{
${ }^{2}$ See d'Espagnat [1] for a careful analysis of Bohr's position.

${ }^{3}$ Actually, the apparatus being in an entangled state with the system has no state by itself strictly speaking since through this entanglement (EPR like), only the system $\mathrm{S}+\mathrm{A}$ has a state. So, it is only a convenient way to speak to say that it is in a superposition of $\left|A_{i}\right\rangle$. The reason why we can safely do that is the fact that the predictions on the apparatus alone (considered as a sub system of $\mathrm{S}+\mathrm{A}$ ) that we can draw from the entangled state of $\mathrm{S}+\mathrm{A}$ are similar to the predictions we could draw from an equivalent superposition of $\left|A_{i}\right\rangle$. The correct formalism in this case is the density matrix that we'll use in the following.
} 
physical universal validity, the consciousness of the observer lies outside of its scope. More precisely, a measurement is made when the consciousness of an observer has an interaction with a system and the interaction between the system and the consciousness of the observer has the physical effect to change the state of the system and to project it according to the reduction postulate. This is the position of Wigner [2] and London and Bauer [3]. Of course, this particular physical effect of a consciousness on a physical system is not very satisfying.

Another possibility is to modify the Schrödinger equation in a way that allows getting a reduction when a measurement is done but preserving of course the current predictions when no measurement is made. The most famous attempt in this direction has been done by Ghirardi, Rimini and Weber [4] (G.R.W. formalism) who add a term in the Schrodinger equation which then describes an evolution from a pure case to a proper mixture. A third possibility is to accept more radical changes by switching to hidden variables theories like the Bohm theory [5] in which the measurement problem doesn't exist.

We don't have time here to examine the pros and cons of these various modified theories and will restrict our analysis of a more recently proposed solution which stays inside the pure quantum formalism. The solution came from a remark from Zeh [6] that no system is really totally isolated. Hence it is necessary to take the environment into account. The decoherence theory is nothing else than the description of the way to take the interaction between the system, the apparatus and the environment into account inside the quantum formalism. We shall first describe briefly the technical framework in which the decoherence theory is usually stated.

\section{The density matrix formalism}

The density matrix formalism has been invented for being able to deal with statistical mixtures of systems being in different pure states, as in classical statistical mechanics. The density matrix of a system in a pure state $\left|\Psi_{S}\right\rangle$ is: $\varrho_{S}=\left|\Psi_{S}\right\rangle\left\langle\Psi_{S}\right|$. For the sake of simplicity, let's take an example in a two dimensional Hilbert space with a basis $\left(\left|\varphi_{1}\right\rangle,\left|\varphi_{2}\right\rangle\right)$ :

Let $\left|\Psi_{S}\right\rangle=\alpha\left|\varphi_{1}\right\rangle+\beta\left|\varphi_{2}\right\rangle\left(\right.$ with $\left.|\alpha|^{2}+|\beta|^{2}=1\right)$ then the density matrix in this basis is:

$$
\varrho_{S}=\left(\begin{array}{cc}
|\alpha|^{2} & \alpha \beta^{*} \\
\alpha^{*} \beta & |\beta|^{2}
\end{array}\right)
$$

In this case: $\varrho_{S}^{2}=\varrho_{S}$

Now imagine a statistical mixture of $\mathrm{N}$ systems in the state $\left|\Psi_{k}\right\rangle$ with probability $p_{k}$

$$
\left|\Psi_{k}\right\rangle=\alpha_{k}\left|\varphi_{1}\right\rangle+\beta_{k}\left|\varphi_{2}\right\rangle
$$

Then $\varrho_{k}=\left|\Psi_{k}\right\rangle\left\langle\Psi_{k}\right|$ and

$$
\rho=\sum_{k} p_{k} \rho_{k}
$$

In this case $\varrho_{S}^{2} \neq \varrho_{S}$

For example, a very simple case of a proper mixture of $\left|\varphi_{1}\right\rangle$ in proportion $\mathrm{N}_{1} / \mathrm{N}=\mathrm{p}_{1}$ and $\left|\varphi_{2}\right\rangle$ in proportion $\mathrm{N}_{2} / \mathrm{N}=\mathrm{p}_{2}$ is represented in the basis $\left(\left|\varphi_{1}\right\rangle,\left|\varphi_{2}\right\rangle\right)$ by:

$$
\varrho_{\mathrm{s}}=\left(\begin{array}{cc}
\mathrm{p}_{1} & 0 \\
0 & \mathrm{p}_{2}
\end{array}\right)
$$

and $\varrho_{s}^{2} \neq$ Qs

This form of the density matrix is analogous to the classical case of a statistical mixture of a proportion $\mathrm{p}_{1}$ of systems in the state $\left|\varphi_{1}\right\rangle$ and a proportion $\mathrm{p}_{2}$ of systems in the state $\left|\varphi_{2}\right\rangle$.

In general, the diagonal element $\rho_{i i}$ is the probability to find the system in the state $\left|\varphi_{i}\right\rangle$ and the 
non-diagonal element $\rho_{i j}$ is linked to the interferences between $\left|\varphi_{i}\right\rangle$ and $\left|\varphi_{j}\right\rangle$.

The Schrödinger equation gives:

$$
i \hbar \frac{d}{d t} \varrho(t)=[H(t), \varrho(t)]
$$

It is important to notice that no individual system can have a diagonal density matrix with more than one non null element. Indeed, as we have seen, if the state of such a system is a superposition of vectors spanning a basis of the Hilbert space $\left|\Psi_{S}\right\rangle=\alpha\left|\varphi_{1}\right\rangle+\beta\left|\varphi_{2}\right\rangle$ then the density matrix is in this basis:

$$
\varrho_{S}=\left(\begin{array}{ll}
|\alpha|^{2} & \alpha \beta^{*} \\
\alpha^{*} \beta & |\beta|^{2}
\end{array}\right)
$$

Whereas if the state of the system is one of the vector of the basis (for example $\left|\varphi_{1}\right\rangle$ ) then the density matrix is:

$$
\varrho_{S}=\left(\begin{array}{ll}
1 & 0 \\
0 & 0
\end{array}\right)
$$

On can see that in neither case the density matrix can be:

$$
\varrho_{S}=\left(\begin{array}{cc}
|\alpha|^{2} & 0 \\
0 & |\beta|^{2}
\end{array}\right)
$$

Of course this is true for any space of higher dimensionality.

So, If we are not in presence of a set of systems initially in different states, such a density matrix describes inevitably what d'Espagnat has called [1] an improper mixture and not a proper mixture. This will be important in the following to show that the decoherence process (that leads to a diagonal density matrix) is not sufficient to explain the reduction of the state vector.

\section{The role of the environment}

Following Zeh's remark, Zurek [7] proposed the following mechanism to explain the reduction. Let's analyze now the measurement process as we did previously but let's take the environment into account and consider a big system composed of the initial measured system plus the apparatus plus the environment.

$$
\varrho_{S A E}=\left|\Psi_{S A E}\right\rangle\left\langle\Psi_{S A E}\right|
$$

After the interaction, according to the Schrödinger equation:

$$
\Psi_{S A E}=\sum c_{i}\left|\varphi_{i}\right\rangle\left|A_{0}\right\rangle\left|E_{0}\right\rangle \rightarrow \sum c_{i}\left|\varphi_{i}\right\rangle\left|A_{i}\right\rangle\left|E_{i}\right\rangle
$$

As previously, we can assume a two dimensional space without loss of generality (and let $c_{1,2}=$ $\alpha, \beta)$. In the basis $\left(\left|\varphi_{1}\right\rangle\left|A_{1}\right\rangle\left|E_{1}\right\rangle,\left|\varphi_{2}\right\rangle\left|A_{2}\right\rangle\left|E_{2}\right\rangle\right)$ we have, similarly to equation (9):

$$
\varrho_{S A E}=\left(\begin{array}{cc}
|\alpha|^{2} & \alpha \beta^{*} \\
\alpha^{*} \beta & |\beta|^{2}
\end{array}\right)
$$

Apparently nothing has been gained! In the basis of the Hilbert space which is the tensorial product of the Hilbert space of the system plus the apparatus plus the environment, the density matrix has exactly the same form than before.

But the key point comes from the remark that we can't perform measurements on all the degrees of freedom of the environment because that would require apparatuses that are totally out of reach. The quantum formalism prescribes in this case that the density matrix of the sub system SA formed by the initial system and the apparatus is given by the partial trace on the degrees of freedom of the environment of $\varrho_{S A E}$ which can be computed as:

$$
\operatorname{Tr}_{E} \varrho_{S A E}=\varrho_{S A}=\left(\begin{array}{cc}
|\alpha|^{2} & Z \alpha \beta^{*} \\
Z \alpha^{*} \beta & |\beta|^{2}
\end{array}\right)
$$

Now, it is possible to show that in general the coefficient $Z(t)$ decreases towards 0 very 
rapidly. So:

$$
\varrho_{S A}(t)=\left(\begin{array}{cc}
|\alpha|^{2} & Z(t) \alpha \beta^{*} \\
Z(t) \alpha^{*} \beta & |\beta|^{2}
\end{array}\right) \rightarrow\left(\begin{array}{cc}
|\alpha|^{2} & 0 \\
0 & |\beta|^{2}
\end{array}\right)
$$

This density matrix looks like the density matrix of the equation (7) that is a statistical mixture and no more like a superposed state. So it seems that each system belonging to the set of systems described by $\varrho_{S A}(t)$ has now a definite state corresponding to one of the eigenvectors of the observable that has been measured. This is the reason why many authors (including Zurek in his first paper) thought that the decoherence process allows to explain in an objective way the reduction of the state vector.

\section{Is the measurement problem solved?}

Solving the measurement problem would mean that, independently of any observer, the initially superposed state of the system has been reduced to a definite state. Now, it's easy to see that it is not the case.

First of all, the final diagonal form of the density matrix is the result of the partial trace of the global density matrix and the reason why this partial trace can be done is entirely due to the fact that it is acknowledged that no measurement of the environment is possible for the observer. It gives the correct predictions provided that the observer won't do any measurement on the environment. That means that the final diagonal form of the density matrix is the form it takes for an observer with limited means of measurement. Hence, it is not an objective reduction. The second reason is that the small non diagonal terms that have been considered as null $(Z(t) \rightarrow 0)$ are actually not rigorously null and can even become again big after a (very) long time.

Another important point to notice is that even though the density matrix looks like the density matrix of a statistical mixture, it is actually the density matrix of an improper mixture. An improper mixture is composed of systems that are all identical. So it is not correct to interpret the decoherence process as leading to a set of systems each having a definite state in a proportion given by the diagonal coefficient of the matrix. The similar form of a matrix got through a partial trace and of the matrix of a proper mixture doesn't allow to assimilate an improper mixture with a proper one. This is particularly visible when one considers only an individual system as we noticed above. As Bell insisted [8], the correct interpretation should be that each system is in a state where all the possibilities are simultaneously present. This is the celebrated "and /or" difficulty.

These reasons show clearly that the decoherence process can't be considered as solving the measurement problem at all. Nowhere is it said what exactly a measurement is and even after decoherence, it is still necessary to use the equivalent of the postulate of reduction and the Born rule to predict what will be observed and with which probability.

What the decoherence process brings is actually an explanation of the classical appearance of the world, provided we use the standard recipes to compute. It explains why we (human observers) can't observe any macroscopic superposition and why what we see is conform to the classical description of the world. But the underlying reality (if there is any) remains in a superposed and entangled state.

If this is taken literally, that means that the reduction postulate is nothing but a convenient and practical way to describe the observations but doesn't correspond to any real physical process. Now, the standard recipes to compute assume that we know what a measurement is. It is when a measurement is made that the probability of finding (observing) a specific result is given by the corresponding diagonal element of the density matrix. But nowhere inside the formalism of 
decoherence it is said what a measurement is. We are left with the initial problem!

\section{What is a measurement?}

Inside a modified theory such as the G.R.W. formalism which changes the Schrodinger equation, a measurement is done through a specific interaction and can be a physical process acting on the state of the system. But if we stay inside the pure quantum formalism, it seems that there is no other way to define a measurement than to say that it is when an observation is made by an observer and even, when a conscious mind becomes aware of the result. The ultimate point is when somebody knows what the result is! That looks like the old proposal of London, Bauer and Wigner. But, if we take the decoherence process into account, there is now a big difference with their position. They thought that the reduction that occurs during a measurement was a physical process through a real action of the mind on the system, the mind changing the state of the system. It is now possible to defend a much less shocking position. The reduction is no more a physical process but merely the fact that when a conscious mind makes an observation what it sees is described by the diagonal density matrix which states that no superposition is visible. That doesn't mean that the state of the system is physically reduced (actually the system remains in an entangled state with the apparatus and the environment) but that what the conscious mind sees can only be conform to a classical appearance as the reduction postulate prescribes. This is reminiscent of the Everett interpretation which says that there is no reduction of the state and that the wave function of the whole universe remains superposed.

\section{The convivial solipsism}

In the Everett interpretation, there is no reduction (the physical world remains in a superposed state) but the observer is divided in as many observers as there are branches (which is not very economical).

If $\left|O_{0}\right\rangle$ is the initial state of the observer and $\left|O_{i}\right\rangle$ is the $\mathrm{i}^{\text {th }}$ state:

$$
\Psi_{S A E O}=\sum c_{i}\left|\varphi_{i}\right\rangle\left|A_{0}\right\rangle\left|E_{0}\right\rangle\left|O_{0}\right\rangle \rightarrow \sum c_{i}\left|\varphi_{i}\right\rangle\left|A_{i}\right\rangle\left|E_{i}\right\rangle\left|O_{i}\right\rangle
$$

We propose another interpretation [9] (extending a first analogous model proposed by d'Espagnat [1]) through a Hanging up mechanism. There is only one observer and one universe but the consciousness of the observer hangs up to one branch. Once the consciousness is hung up to one branch, it will hang up only to branches that are daughters of this branch for all the following observations. That guarantees:

- That repeating the same measurement will give again the same result

- Any conflict with another observer is impossible

Assume for example that the system is a spin half particle in a superposed state along Oz.

$$
\left|\Psi_{S}\right\rangle=\alpha|+\rangle_{z}+\beta|-\rangle_{z}
$$

After the interaction with the apparatus and taking the environment into account the global state is:

$$
\left|\Psi_{S A E}\right\rangle=\alpha|+\rangle_{z}|\uparrow\rangle\left|E_{+}\right\rangle+\beta|-\rangle_{z}|\downarrow\rangle\left|E_{-}\right\rangle
$$

If we include the state of the observer we get:

$$
\left|\Psi_{S A E}\right\rangle=\alpha|+\rangle_{z}|\uparrow\rangle\left|E_{+}\right\rangle|\odot\rangle+\beta|-\rangle_{z}|\downarrow\rangle\left|E_{-}\right\rangle|\otimes\rangle
$$

Now, the hanging up mechanism says that the consciousness of the observer chooses one branch at random (respecting the Born rule linked to the coefficients of the linear combination). 
Hence:

$$
\left|O_{0}\right\rangle->\mid \odot>>\text { or }\left|O_{0}\right\rangle->\mid \otimes>
$$

So, even if the universal superposed wave function is not reduced, for all subsequent measurements for this observer everything happens as if the wave function was reduced to either $|+\rangle_{z}|\uparrow\rangle\left|E_{+}\right\rangle|\odot\rangle$ or $|-\rangle_{z}|\downarrow\rangle\left|E_{-}\right\rangle|\otimes\rangle$.

Nevertheless, this observer continues to physically exist in the other branches even if he can't be conscious of what happens in these branches.

That is a sort of solipsism because the consciousness of each observer is located inside its own branch independently of the others. But that is convivial since no conflict is possible. The quantum rules and the hanging up mechanism for each observer prevent any possibility to notice a divergence between the perceptions of two different observers.

There is another striking consequence that is a strange answer to the famous phrase of Einstein: God doesn't play dice. In this case, Einstein was right, God doesn't play dice but you do! This is so because the random aspect of the quantum predictions comes, not from the fact that the physical systems changes at random (the dynamic of the Universe is fully deterministic) but from the random way your consciousness chooses the branch to which it hangs up!

\section{References}

[1] B. d'Espagnat, Le Réel voilé, analyse des concepts quantiques, Fayard, 1994. English Transl. (2003), Veiled Reality: An Analysis of Quantum Mechanical Concepts, 2003, Westview Press, Boulder, Colorado.

[2] E.P. Wigner, Symetries and Reflections, Bloomington, Indiana University Press, 1967.

[3] F. London, E. Bauer, La théorie de l'observation en mécanique quantique, Hermann, 1939.

[4] G.C. Ghirardi, A. Rimini, T. Weber, Phys. Rev., D34, 470, 1986.

[5] D. Bohm, Quantum Theory, Prentice-Hall, 1951.

[6] H. Zeh, Foundations of Physics, 1, 67, 1970.

[7] W. Zurek, Phys. Rev. D24, 1516, 1981 and Phys. Rev. D26, 1862, 1982.

[8] J.S. Bell, Against Measurement, in Sixty Two Years of Uncertainty, A.I. Millered., New York, Plenum Press, 1990.

[9] H. Zwirn, les limites de la connaissance, Odile Jacob, 2000. 